\title{
Characterizing Dense Nanosuspensions using Cryo-SEM
}

${ }^{1}$ Nazia N. Khawaja, ${ }^{2}$ David J. Goldfarb, ${ }^{3}$ Sachin Mittal, ${ }^{3}$ Irina Kazakevich, and ${ }^{1}$ Matthew Lamm

1. Merck Research Laboratories, Preformulation Sciences, Rahway, NJ

2. Merck Manufacturing Division, Center for Materials Sciences \& Engineering, Rahway, NJ

3. Merck Research Laboratories, Sterile Formulation Sciences, Kenilworth, NJ

Cryogenic-Scanning Electron Microscopy (cryo-SEM) is a technique which unlike standard SEM, allows the imaging of samples in their hydrated state. This technique was used to image dense nanosuspensions. Standard SEM images were captured previously, however, were questionable in value, because the sample needed to be dried prior to measurement. There were concerns that the particles could be altered upon drying, especially in the presence of surfactant. By using the cryo-SEM technique, this drying step was eliminated and the particles were able to be viewed in situ. The nanosuspension particle morphology, imaged with a Hitachi SU 5000 scanning electron microscope equipped with a Quorum PP3010T cryo unit, correlated with particle size measurements obtained with an orthogonal Dynamic Light Scattering (DLS) technique. It was also previously shown for these nanosuspensions that rheological measurements correlated with median particle size as measured by DLS.

The DLS measurements for one nanosuspension formulation exhibited a median particle size growth and a corresponding increase in viscosity during accelerated stability studies. Two theories were hypothesized as to the cause for this phenomenon: aggregation of particles or Ostwald ripening. With the availability of cryo-SEM images, aggregation was ruled out. It is suspected that the morphology and size differences between these batches, observed by SEM and DLS, resulted in an increased viscosity (Figure 1). In general, cryo-SEM was able to provide unique images that could not have been produced using traditional microscopy techniques, proving to be a useful tool for viewing nanoparticles in situ. 


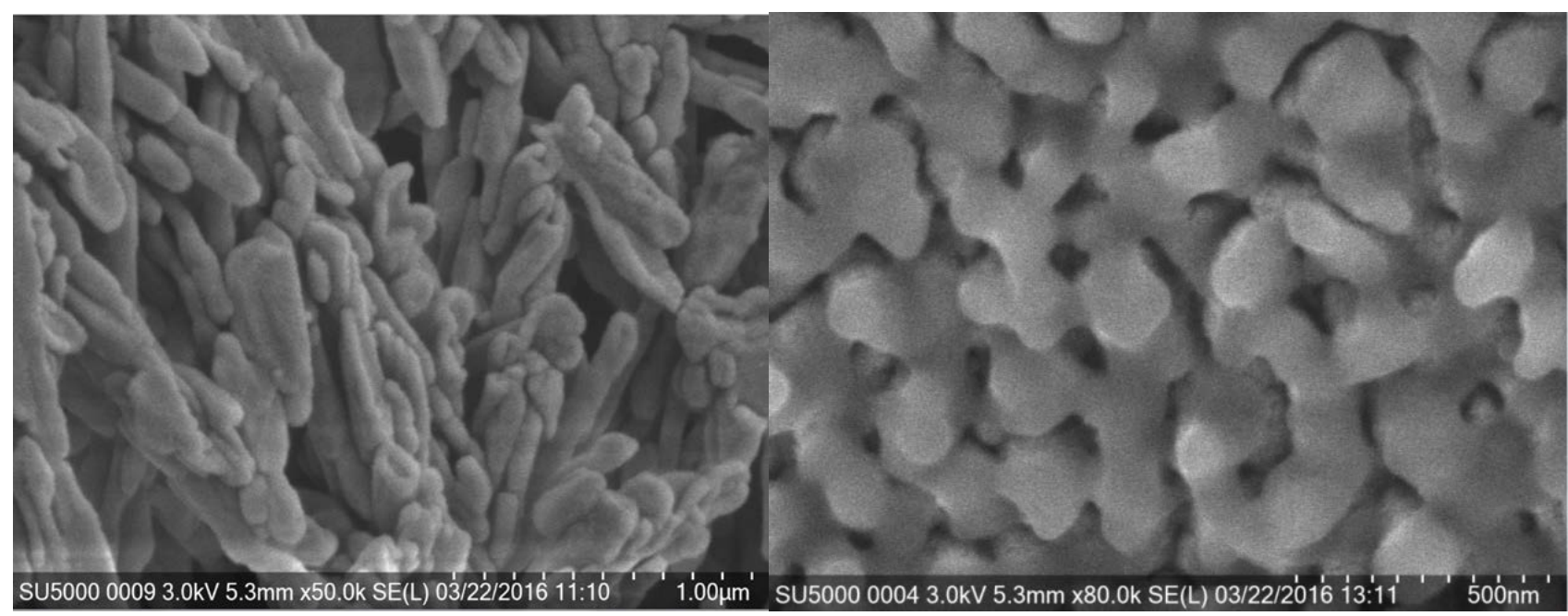

Figure 1: Cryo-SEM images of a batch which pasted ( left) versus a batch which was a free flowing nanosuspension ( right). A large presence of rods can be observed in the batch which pasted indicating that aspect ratio may play a role in the rheological properties of the nanosuspension. 